\title{
Gestión de la Calidad y del Medio Ambiente en Instituciones de Educación Superior mediante Integración de ISO 9001 e ISO 14001
}

\author{
Diego A. Tlapa, Jorge Limón y Yolanda A. Báez \\ Universidad Autónoma de Baja California, Facultad de Ingeniería Ensenada, Carretera Tijuana- \\ Ensenada Km. 103, Ensenada, Baja California-México (e-mail: diegotlapa@uabc.mx)
}

\begin{abstract}
Resumen
El presente trabajo tiene por objetivo describir la situación de los sistemas de gestión de la calidad y del medio ambiente en instituciones de educación superior en México, así como analizar la factibilidad de la integración de gestión de la calidad y medio ambiente. Para ello, se realizó una búsqueda de instituciones de educación superior que trabajan bajo ISO 9001 e ISO 14001, encontrando 322 y 3 certificados respectivamente en el año 2008. El trabajo propone además un grupo de procedimientos integrados aplicables a las instituciones de educación superior, que cuenten o contarán con un sistema de gestión de la calidad y buscan la forma de gestionar el ambiente de una manera práctica. Se concluye que es factible la integración de los requisitos de un sistema de gestión ambiental a los de uno de gestión de la calidad.
\end{abstract}

\section{Quality and Environmental Management in Higher Education Institutes by Integrating ISO 9001 and ISO 14001}

\begin{abstract}
The goal of this work is to describe the situation of quality and environmental management systems situation in higher education institutions in Mexico and to analyze the integration of quality and environmental management. For this, a database with all higher education institutions in Mexico that deals with ISO 9001 and ISO 14001, was done. A total of 322 and 3 certificates respectively in 2008 were found. It is proposed an integrated group of procedures applicable at higher education institutes which have or will have a quality management system and that are looking for a practical environmental management system. It is concluded that an integration of environmental management system to a quality management system is feasible.
\end{abstract}

Key words: quality management system, environmental management system, ISO 9001, ISO 14001, higher education 


\section{INTRODUCCIÓN}

A nivel mundial el auge por la gestión ambiental llegaba en el año 2007 a 154,572 organizaciones, en general certificadas bajo ISO 14001, mientras que en gestión de la calidad se contabilizaba un total de 951,486 bajo ISO 9001 en 161 países (ACNielsen, 2009); es decir, más de un millón de organizaciones certificadas con ambos estándares y con una proyección de aumento de certificados en organizaciones de países en vías de desarrollo. En este contexto y en un entorno cada vez más competitivo, las Instituciones de Educación Superior (IES) enfrentan retos importantes, como la necesidad de gestionar de forma eficaz y eficiente sus diferentes actividades y recursos, por lo que demandan a su vez modelos de sistemas de gestión que puedan implementar de manera practica, como el estándar ISO 9001, el cual plantea una serie de requerimientos genéricos para la implementación de un sistema de gestión de la calidad.

Se puede mencionar que no hay un consenso universal acerca de la mejor manera de gestionar las iniciativas de mejora de la calidad dentro de instituciones de educación superior (Becket y Brookes, 2006). Para la industria el desarrollo de la gestión de la calidad surgió desde adentro; en contraste, la calidad imperante en la educación superior viene de afuera y no esta basada en la necesidad de mejora, menciona Houston (2007), sino por la presión del mercado y del gobierno. Al respecto Cidad (2004), menciona que la universidad debe actuar con mayor ambición estratégica y superior autoestima organizacional, planteándose como objetivo la incorporación de la cultura y el dispositivo que le permita superar la implantación y mantenimiento de un SGC.

Cidad (2004), comenta que la implantación de un SGC en una organización encallecida, como suele ser el caso de la universidad, supone una oportunidad de modernización y liberación del lastre de la no calidad, largo tiempo acumulado; lastre que se manifiesta en forma de abundantes prácticas que no añaden valor a los procesos o que los obstaculizan, constituyendo auténtica basura organizacional. El principal reto para adaptar los principios de la gestión total de la calidad en la educación superior es la necesidad de lidiar con la intangibilidad del proceso educacional (Dounos y Bohoris, 2007). La gestión de la calidad es pues una necesidad, sin embargo al ser un ejemplo para la sociedad las IES se enfrentan también en la necesidad de demostrar un desempeño ambiental adecuado, y deben predicar con el ejemplo. En este sentido varias de ellas han adoptado o comienzan por implementar el sistema de gestión ambiental que propone el estándar ISO 14001. Ortiz y Nieto (2003), indican que posiblemente el punto de mayor tensión en el futuro es el que ubica a las IES como actores sociales estratégicos y protagónicos en la búsqueda de la sustentabilidad, a través del conocimiento, de un sentido solidario y de justicia social intergeneracional, por su parte Ortega (2003), indica que predicar con el ejemplo es una estrategia a seguir por las IES, y se refiere al desempeño ambiental.

Cuando las IES gestionan la calidad bajo ISO 9001, también pueden gestionar el medio ambiente bajo ISO 14001, ya sea de manera separada o integral, sin embargo cuando se realiza de manera separada, se puede realizar trabajo repetido; hoy por hoy la tendencia es la simplificación, ¿por qué contar con dos o más sistemas de gestión de manera independiente en las IES?, esta pregunta surge de manera natural, ya que esto implica procedimientos y documentación por separado y por ende más costo y burocracia, cuando en la actualidad los estándares ISO 9001 e ISO 14001 presentan estructuras similares que facilitan la integración de los sistemas resultantes en un solo Sistema Integral de Gestión (SIG). Ante esta situación, las IES que cuentan ya con un SGC bajo ISO 9001, tienen una excelente oportunidad para integrar a éste el SGA de ISO 14001. Renzi y Cappelli (2000), indican que para lograr una valorización máxima de dichos sistemas, se debe utilizar aspectos comunes en ambos estándares, entonces incluso las desventajas pueden ser oportunidades.

Una de los principales dificultades que enfrentan las IES, es la forma en que deben gestionar de manera practica y simultanea la calidad, así como el cuidado del medio ambiente al realizar sus propias actividades; por lo tanto, se considera oportuno exponer algunas ideas, datos, reflexiones y recomendaciones relacionadas con el estado actual de los sistemas de gestión en IES en México y la forma de integrar los requisitos de los estándares internacionales ISO 9001 e ISO 14001 respectivamente. Lo anterior con el objetivo de contribuir a la comunidad en general y en especial a las IES con información sobre la gestión de la calidad y del medio ambiente. 


\section{GESTIÓN DE LA CALIDAD, GESTIÓN AMBIENTAL Y DESARROLLO SUSTENTABLE EN LA EDUCACIÓN SUPERIOR, CASO MÉXICO}

La educación es un factor clave que deberá contribuir al mejoramiento de la calidad de vida en todas sus dimensiones, de ahí que sea impostergable desarrollar un sistema para que los educandos sean formados en un ambiente de calidad, que les brinde la oportunidad efectiva de desarrollar competencias intelectuales, técnicas y valorativas a la altura de las exigencias del nuevo entorno (Consejo de especialistas para la educación, 2006); lo anterior plantea la necesidad que el proceso de enseñanza-aprendizaje y los de apoyo en instituciones de educación busquen mejorar continuamente la calidad de los mismos y en este sentido el estándar ISO 9001 es una opción que las IES han tomado y que se puede apoyar en la guía de implementación en la educación IWA 2.

En el 2002, mediante la resolución 57/254, la Asamblea General de la ONU adoptó el Decenio de las Naciones Unidas para la Educación con miras al Desarrollo Sustentable (2005-2014) y designó a la UNESCO para promoverlo. Esta iniciativa hace un llamado a los gobiernos para establecer una sólida plataforma institucional y social, además de proponer alianzas en todos los niveles, comenzando en el ámbito local, hasta cubrir el contexto nacional e internacional. Dos de los retos que deben asumir las universidades en su papel de promotoras de la sustentabilidad, son la congruencia, dar el ejemplo desde el interior de los campus; y la pertinencia, incluir la sustentabilidad en sus funciones esenciales enfatizando su compromiso social (Complexus, 2006). En este sentido ISO 9001 e ISO 14001 pueden ayudar a incorporar la sustentabilidad en los procesos de una IES.

La sustentabilidad plantea 3 ejes principales: medio ambiente, social y económico. Al gestionar la calidad de los procesos de una IES, se impacta socialmente al tener mejores servicios educativos; cuando más estudiantes interactúan con procesos que buscan la mejora continua, similares a los del sector productivo, se contribuye a la cultura de la calidad que tanto necesitan las organizaciones y que impacta en el aspecto económico de los países; mientras que el aspecto ambiental puede ser cubierto al gestionar el medio ambiente tal como lo plantea ISO 14001. Al respecto, la globalización y los tratados internacionales han incentivado la adopción de este estándar en los diferentes países, más aún cuando los destinos para la exportación de esos países también lo han implementado (Potoski y Prakash, 2004); y es que la adopción de ISO 14001 esta siendo más rápida en comparación al estándar ISO 9001 cuando éste fue lanzado, esto debido a que la experiencia lograda por las organizaciones con ISO 9001, removió parte de la incertidumbre acerca del valor y relevancia de los estándares de sistemas de gestión (Alburquerque et al., 2007). En este contexto se prevé que cada vez más IES en México comiencen a implementar el sistema de gestión ambiental que requiere el estándar internacional ISO 14001, el cual en 2008 contabilizaba 3 certificados.

En el año 2005, la Secretaría de Educación Pública (SEP) y la Secretaría de Medioambiente y Recursos Naturales (SEMARNAT), suscribieron un compromiso para promover la educación como base para transitar hacia una sociedad humana sustentable y que actúa en tres áreas básicas:

1. La reorientación de los programas educativos, desde preescolar hasta la educación superior, a fin de impulsar los principios, conocimientos, habilidades, perspectivas y valores relacionados con la sustentabilidad.

2. El desarrollo de la comprensión y la conciencia pública sobre la importancia de la sustentabilidad en todos los aspectos de la vida cotidiana.

3. La operación de programas de capacitación para asegurar que el personal de todos los sectores productivos del país, posee el conocimiento y habilidades necesarias para desempeñar su trabajo de manera sustentable.

A su vez la Asociación Nacional de Universidades e Instituciones de Educación Superior (ANUIES) propone diferentes líneas de trabajo para la gestión ambiental, incluyendo las de carácter institucional, que corresponden a las acciones que deben realizar directamente las instituciones de educación superior (Ortega, 2003). Bajo este contexto cada IES puede gestionar 
el medio ambiente al implementar ISO 14001 y esta implementación será más fácil sí ya cuenta con la gestión de la calidad bajo ISO 9001, ya que ambas normas presentan una excelente oportunidad para integrarse en un solo sistema integral de gestión.

\section{ESTRATEGIAS DE INTEGRACIÓN DE ISO 9001 E ISO 14001}

Para De-Olivera y Coelho (2002), la integración de sistemas, es un cambio más sencillo cuando las cosas no han funcionado bien. Sin embargo, la decisión de integrar o no sistemas de gestión, esta enfocada sobre los intereses de la organización, por lo que se debe determinar una estrategia para lograr que la integración realmente suceda. Cuando esta estrategia es débil, el resultado pudiera ser políticas paralelas, descontento interno y externo a la organización, y desperdicios de recursos Wilkinson y Dale (1999), encuentran que un Sistemas Integral de Gestión (SIG) resulta cuando dos o más sistemas de gestión están ligados de tal manera que la independencia de uno está perdida. A su vez plantean que la integración de sistemas de gestión puede tomar lugar en diferentes maneras o niveles como se muestran a continuación:

Nivel 1. Involucra la integración en toda la organización del sistema de gestión de la calidad (SGC) a la norma ISO 9001.

Nivel 2. La integración se da sobre una combinación de elementos comunes entre un SGC y un SGA.

Nivel 3. Esta Integración involucra unir SGC y SGA con otros sistemas de gestión.

Nivel 4. Involucra la integración de todos los sistemas de gestión aplicables a la organización, lo que sugiere un verdadero SIG.

Para el logro de la integración Kit-Fai y Ip-Kee (2002), encuentran algunas características clave como son: el compromiso de la dirección, el entrenamiento, educación del personal, el control de documentos y las auditorias. González (2006), indica que los SIG, permiten a las organizaciones perfeccionar su trabajo desde el punto de vista de los procesos estratégicos, clave y de apoyo. Por su parte Karapetrovic y Willborn (1998), ofrecen 3 estrategias para la integración nivel 2, es decir, la resultante de integrar un sistema de gestión de la calidad y uno de gestión ambiental bajo ISO 9001 e ISO 14001:

1. Implementar primeramente un sistema de gestión de la calidad bajo ISO 9001 y posteriormente integrar a éste un sistema de gestión ambiental bajo ISO 14001. En estos casos se recomienda que madure el SGC más de un año y después buscar la integración (ver figura 1).

2. Implementar primeramente un sistema de gestión ambiental bajo ISO 14001 y posteriormente integrar un sistema de gestión de la calidad bajo ISO 9001. Al igual que el anterior, se recomienda dejar un año para que madure el SGA (ver figura 2).

3. Implementación simultanea del sistema de gestión de la calidad y ambiental, e integrando ambos en uno solo sistema. Esta estrategia requiere mayor atención y conocimiento en un inicio, y que, al no tener ninguna referencia anterior en SG, hace más complicada la integración (ver figura $3)$.

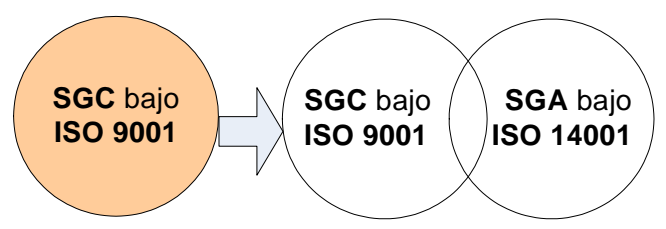

Fig. 1: Estrategia de integración basada en un SGC.

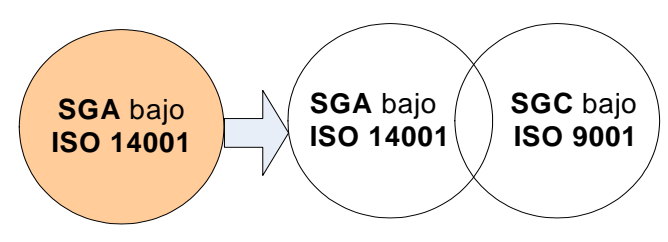

Fig. 2: Estrategia de integración basada en un SGA. 


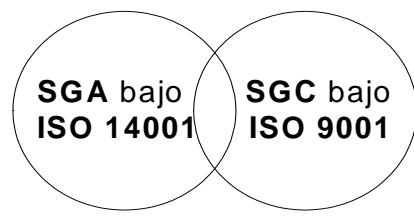

Fig. 3: Estrategia de implementación e integración simultanea.
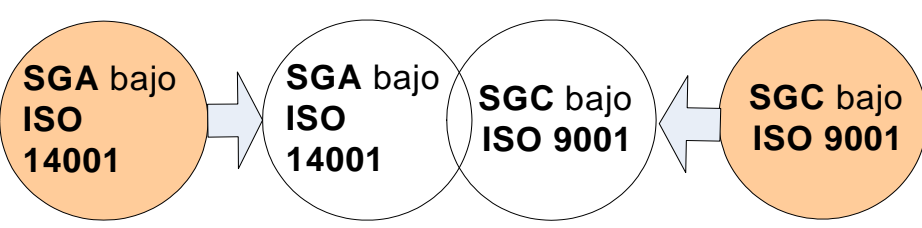

Fig. 4: Estrategia de integración en sistemas de gestión ya implementados.

En IES que ya cuenten con los 2 sistemas de gestión, pero que trabajan separadamente, se facilita la integración debido al conocimiento y experiencia adquirida. En estos casos lo más recomendable es integrar ambos SG y evitar redundancias de procedimientos similares (ver figura 4).

Para Renzi y Cappelli (2000), la integración tiene una serie de ventajas como: reducción de costos por que se mejora la administración de las personas y de los datos, homogeneidad en la metodología de administración, y reducción de documentación con la creación de formas comunes que pueden ser usadas por más personas. Por su parte Shaw (2004), indica tres razones principales para combinar estándares en un sistema integral de gestión: evitar la duplicación de esfuerzos, facilidad de uso de un solo arreglo que se usa frecuente y es más familiar que de manera desconectada, y reducción de costo.

\section{Otras ventajas pueden ser:}

1 Facilita la planificación integral.

2 Facilita la asignación de recursos.

3 Ayuda al establecimiento de objetivos complementarios.

4 Facilita la evaluación de la eficacia global de una IES.

5 Facilidad para auditar el sistema de gestión contra los requisitos de ISO 9001 e ISO 14001.

6 Una única norma ISO 19011 que unifica los requisitos de auditoria para SGC y SGA.

7 Reducción de tiempos de auditorias.

Dificultades en la integración de sistemas de gestión.

1 Es relativamente nuevo ISO 14001 para algunas IES.

2 Implica contar con personal capacitado en SGC y SGA.

3 No se cuenta con certificado para ambas normas integradas, solo por separado.

4 Mayor costo de certificación donde se desee contar con los 2 certificados.

En un estudio sobre SIG en el Reino Unido, Douglas y Glen (2000), refieren algunos beneficios sobre una muestra de 28 organizaciones con sistemas integrados de gestión, en la que más de la mitad de los encuestados manifiestan beneficios observados con la implementación de un SIG, un $71 \%$ manifiesta que experimentaron reducción de costos y un $82 \%$ de tales organizaciones experimentaron mayor efectividad interna y externa, ver Tabla 1.

Tabla 1: Beneficios con sistemas integrados de gestión.

\begin{tabular}{|l|l|c|c|c|}
\hline \multirow{2}{*}{} & \multicolumn{2}{|c|}{ SI } & \multicolumn{2}{c|}{ NO } \\
\cline { 2 - 6 } & $\%$ & cantidad & $\%$ & cantidad \\
\hline Menos procedimientos & 82 & 23 & 18 & 5 \\
\hline Menos burocracia & 86 & 24 & 14 & 4 \\
\hline Auditores multifuncionales & 89 & 25 & 11 & 3 \\
\hline Facilidad para manejar SG & 82 & 23 & 18 & 5 \\
\hline Mayor efectividad-interna y externa & 82 & 23 & 18 & 5 \\
\hline Mejor comunicación entre el Staff & 61 & 17 & 39 & 11 \\
\hline Mejoramiento de la imagen con los clientes & 50 & 14 & 50 & 14 \\
\hline Reducción de costos & 71 & 20 & 29 & 8 \\
\hline
\end{tabular}




\section{METODOLOGÍA}

Estado de ISO 9001 e ISO 14001 en IES. Para conocer el estado y cantidad de certificados de ISO 9001 e ISO 14001 en IES, se realizó una búsqueda de información en los sitios electrónicos y por solicitud directa a las 35 casas certificadoras que operan en México en el sector educativo según la Entidad Mexicana de Acreditación (EMA). Los resultados de la búsqueda representan la cantidad de certificados hasta Noviembre 2008 y son presentados en la sección de resultados.

Nivel de integración de ISO 9001 e ISO 14001. En la búsqueda de la integración de los sistemas de gestión, se analiza el estándar ISO 9001:2008 (2001), el cual presenta en esta nueva edición 137 requisitos distribuidos en 51 cláusulas, mientras que el estándar ISO 14001:2004 (2004) presenta 62 requisitos en 18 cláusulas. Todos los requisitos deberán cumplirse a excepción de algunas posibles exclusiones del capítulo 7 de ISO 9001, dependiendo el alcance del sistema. Para una correcta integración es importante resaltar que los requisitos en ambos estándares se pueden agrupar en tres categorías:

1. Requisitos similares; presentan la misma estructura y solo varia en la orientación a la calidad y medio ambiente. De tal manera que al cumplir con ISO 9001 implica la base para cumplir con ISO 14001; un sólo procedimiento orientado a cubrir los 2 estándares es suficiente, lo que evita el doble control. Algunos de estos requisitos son los procedimientos de: control de documentos, control de registros, auditoria interna, revisión por la dirección y competencia, formación y toma de conciencia. Por ejemplo, al contar con un procedimiento de control de documentos para el SGC, éste se modifica para que abarque el control de documentos para la gestión ambiental, dando como resultado un único procedimiento de control de documentos.

2. Requisitos compatibles; estos requisitos en ambos estándares son afines y presentan una independencia menor, lo que facilita la integración bajo un esquema general. Un ejemplo de requisito compatible es la política ambiental y política de la calidad, las cuales se pueden integrar en una sola política integral de gestión.

Los requisitos similares y compatibles encontrados en ambos estándares se enlistan en la Tabla 2 y en la Tabla 3.

3. Requisitos específicos; algunos requisitos de ambos estándares no presentan una relación fuerte lo que se identifica como requisitos específicos a cada estándar y que se manejan por separado. Algunos requisitos específicos son: procedimiento de respuesta ante emergencias y evaluación del cumplimiento legal. Por ejemplo Respuesta ante emergencias es un procedimiento que se considera específico a ISO 14001 y no se integra a otro procedimiento de ISO 9001, sin embargo se encuentra referenciado en el resultante manual de gestión integral.

Procedimientos integrales de gestión. En ambas normas se requieren ciertos procedimientos a elaborar donde se especifica como una IES Ileva cierta actividad; Para De-Olivera y Coelho (2002), una organización debe ajustar su sistema de gestión a su dimensión y realidad, solo documentando lo que estrictamente es necesario y así evitar la sobre-documentación y burocracia. Referente a la norma ISO 9001:2008 exige 6 procedimientos obligatorios, por su parte la norma ISO 14001:2004 presenta 14 procedimientos de carácter similar. Partiendo de estos procedimientos obligatorios se analizan requisitos comunes en ellos para identificar cuáles son los procedimientos integrales que cubran ambos estándares.

Cabe señalar que ISO 14001 no exige el desarrollo de un manual de gestión ambiental, pero ISO 9001 si, por lo que se propone como base para unir la documentación de ambos estándares.

\section{RESULTADOS}

Estado de ISO 9001 e ISO 14001 en IES. Los resultados de la búsqueda de certificados tanto de ISO 9001 como de ISO 14001, arrojó que para finales de 2008 existían en México 322 certificados de SGC bajo ISO 9001 en IES y solo 3 certificados de SGA bajo ISO 14001, lo cual presenta una gran diferencia entre ambos, y es que el fenómeno de la gestión de la calidad en la educación 
superior en México crece fuertemente y tiene impacto en los estudiantes de licenciatura, que en el país representan 2, 528,664 estudiantes para el periodo 2006-2007 (Suárez, 2007). Sin embargo la certificación también representa para las IES una forma de hacerse llegar de recursos Federales como el Programa Integral de Fortalecimiento Institucional (PIFI). En el caso de la Universidad Autónoma de Baja California, para el periodo 2007 recibió \$3,784,412 m.n. (343,101 Dólares estadounidenses) para asegurar la calidad de los 121 procesos de gestión y laboratorios certificados con la norma ISO 9001 a través de capacitación, auditoria, certificación entre otros.

Tabla 2: Requisitos similares y compatibles entre ISO 9001 e ISO 14001.

\begin{tabular}{|c|c|c|c|c|c|}
\hline \multicolumn{3}{|c|}{ ISO 9001:2008 } & \multicolumn{3}{|c|}{ ISO 14001:2004 } \\
\hline Requisito & Descripción & Elen & ento & Descripción & Requisito \\
\hline 1 & Requisitos generales & 4.1 & 4.1 & Requisitos generales & 1 \\
\hline 1 & Generalidades & 4.2 .1 & 4.4 .4 & Documentación & 1 \\
\hline 3 & Control de documentos & 4.2 .3 & 4.4 .5 & Control de documentos & 3 \\
\hline 3 & Control de registros & 4.2 .4 & 4.5 .4 & Control de registros & 3 \\
\hline 1 & Enfoque al cliente & 5.2 & 4.3 .1 & Aspectos ambientales & 1 \\
\hline 1 & Política de calidad & 5.3 & 4.2 & Política ambiental & 1 \\
\hline 2 & Objetivos de la calidad & 5.4 .1 & 4.3 .3 & $\begin{array}{l}\text { Objetivos, } \\
\text { programas }\end{array}$ & 3 \\
\hline 1 & $\begin{array}{l}\text { planificación del sistema de } \\
\text { gestión de la calidad }\end{array}$ & 5.4 .2 & 4.3 .3 & $\begin{array}{lll}\begin{array}{l}\text { Objetivos, } \\
\text { programas }\end{array} & \text { metas } & \mathrm{y} \\
\end{array}$ & 2 \\
\hline 1 & $\begin{array}{l}\text { Responsabilidad } \quad y \\
\text { autoridad }\end{array}$ & 5.5 .1 & 4.4 .1 & $\begin{array}{l}\text { Recursos, } \\
\text { responsabilidad y autoriones, }\end{array}$ & 1 \\
\hline 2 & $\begin{array}{lll}\text { Representante } & \text { de } & \text { la } \\
\text { dirección } & & \end{array}$ & 5.5 .2 & 4.4 .1 & $\begin{array}{l}\text { Recursos, funciones, } \\
\text { responsabilidad y autoridad }\end{array}$ & 2 \\
\hline 1 & Comunicación interna & 5.5 .3 & 4.4 .3 & Comunicación & 1 \\
\hline 3 & Generalidades & 5.6 .1 & 4.6 & Revisión por la dirección & 3 \\
\hline 1 & Información para la revisión & 5.6 .2 & 4.6 & Revisión por la dirección & 1 \\
\hline 1 & Resultados de la revisión & 5.6 .3 & 4.6 & Revisión por la dirección & 1 \\
\hline 1 & Provisión de recursos & 6.1 & 4.4 .1 & $\begin{array}{l}\text { Recursos, funciones, } \\
\text { responsabilidad y autoridad }\end{array}$ & 1 \\
\hline 1 & Generalidades & 6.2 .1 & 4.4 .2 & $\begin{array}{l}\text { Competencia, formación y } \\
\text { toma de conciencia }\end{array}$ & 1 \\
\hline 1 & $\begin{array}{l}\text { Competencia, toma } \\
\text { conciencia y formación }\end{array}$ & 6.2 .2 & 4.4 .2 & $\begin{array}{l}\text { Competencia, formación } \mathrm{y} \\
\text { toma de conciencia }\end{array}$ & 5 \\
\hline 1 & Infraestructura & 6.3 & 4.4 .1 & $\begin{array}{l}\text { Recursos, } \\
\text { responsabilidad y autoridad }\end{array}$ & 1 \\
\hline 1 & $\begin{array}{lll}\begin{array}{l}\text { Determinación } \\
\text { requisitos }\end{array} & \text { de } & \text { los } \\
\end{array}$ & 7.2 .1 & $\begin{array}{l}4.3 .1 \\
4.3 .2\end{array}$ & $\begin{array}{l}\text { Aspectos ambientales } \\
\text { Requisitos legales y otros }\end{array}$ & 2 \\
\hline 3 & 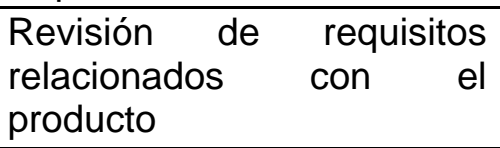 & 7.2 .2 & 4.3 .1 & Aspectos ambientales & 2 \\
\hline 1 & Comunicación con el cliente & 7.2 .3 & 4.4 .3 & Comunicación & 1 \\
\hline 3 & $\begin{array}{l}\text { Planificación del diseño y } \\
\text { desarrollo }\end{array}$ & 7.3 .1 & 4.4 .6 & Control operacional & 1 \\
\hline 3 & $\begin{array}{l}\text { Elementos de entrada para } \\
\text { diseño y desarrollo }\end{array}$ & 7.3 .2 & 4.4 .6 & Control operacional & 1 \\
\hline 2 & $\begin{array}{l}\text { Resultados del diseño y } \\
\text { desarrollo }\end{array}$ & 7.3 .3 & 4.4 .6 & Control operacional & 1 \\
\hline 1 & $\begin{array}{c}\text { Revisión del diseño y } \\
\text { desarrollo }\end{array}$ & 7.3 .4 & 4.4 .6 & Control operacional & 1 \\
\hline
\end{tabular}




\begin{tabular}{|c|c|c|c|c|c|}
\hline 1 & $\begin{array}{c}\text { Verificación del diseño y } \\
\text { desarrollo }\end{array}$ & 7.3 .5 & 4.4 .6 & Control operacional & 1 \\
\hline 1 & $\begin{array}{c}\text { ValidaciÔn del diseÒo y } \\
\text { desarrollo }\end{array}$ & 7.3 .6 & 4.4 .6 & Control operacional & 1 \\
\hline 2 & $\begin{array}{c}\text { Control de los cambios del } \\
\text { diseño y desarrollo }\end{array}$ & 7.3 .7 & 4.4 .6 & Control operacional & 1 \\
\hline 4 & Proceso de compras & 7.4 .1 & 4.4 .6 & Control operacional & 1 \\
\hline 2 & Información de las compras & 7.4 .2 & 4.4 .6 & Control operacional & 1 \\
\hline
\end{tabular}

Tabla 3: Requisitos similares y compatibles entre ISO 9001 e ISO 14001 (continuación).

\begin{tabular}{|c|c|c|c|c|c|}
\hline \multicolumn{3}{|c|}{ NMX-CC-9001-IMNC-2000 } & \multicolumn{3}{|c|}{ NMX-SAA-14001-IMNC-2004 } \\
\hline Requisito & Descripción & Ele & nto & Descripción & Requisito \\
\hline 4 & Proceso de compras & 7.4 .1 & 4.4 .6 & Control operacional & 1 \\
\hline 2 & Información de compras & 7.4 .2 & 4.4 .6 & Control operacional & 1 \\
\hline 1 & $\begin{array}{l}\text { Verificación de los } \\
\text { productos comprados }\end{array}$ & 7.4 .3 & 4.4 .6 & Control operacional & 1 \\
\hline 2 & $\begin{array}{l}\text { Control de producción y } \\
\text { prestación del servicio }\end{array}$ & 7.5 .1 & 4.4 .6 & Control operacional & 1 \\
\hline 2 & $\begin{array}{c}\text { Validación de los } \\
\text { procesos de la producción } \\
\text { y la prestación del } \\
\text { servicio }\end{array}$ & 7.5 .2 & 4.4 .6 & Control operacional & 1 \\
\hline 5 & $\begin{array}{c}\text { Control de los dispositivos } \\
\text { de seguimiento y } \\
\text { medición }\end{array}$ & 7.6 & 4.5 .1 & Seguimiento y medición & 4 \\
\hline 1 & Generalidades & 8.1 & 4.5 .1 & Seguimiento y medición & 1 \\
\hline 6 & Auditoria interna & 8.2 .2 & 4.5 .5 & Auditoria interna & 5 \\
\hline 1 & $\begin{array}{l}\text { Seguimiento y medición } \\
\text { de los procesos }\end{array}$ & 8.2 .3 & $\begin{array}{l}4.5 .1 \\
4.5 .2\end{array}$ & $\begin{array}{l}\text { Seguimiento y medición } \\
\text { Evaluación del } \\
\text { cumplimiento legal }\end{array}$ & $\begin{array}{l}1 \\
1\end{array}$ \\
\hline 2 & $\begin{array}{c}\text { Seguimiento y medición } \\
\text { del producto }\end{array}$ & 8.2 .4 & $\begin{array}{l}4.5 .1 \\
4.5 .2\end{array}$ & $\begin{array}{l}\text { Seguimiento y medición } \\
\text { Evaluación del } \\
\text { cumplimiento legal }\end{array}$ & $\begin{array}{l}2 \\
1\end{array}$ \\
\hline 1 & Análisis de datos & 8.4 & 4.5 .1 & Seguimiento y medición & 1 \\
\hline 3 & Acción correctiva & 8.5 .2 & 4.5.3 & $\begin{array}{c}\text { No conformidad, acción } \\
\text { correctiva y acción } \\
\text { preventiva }\end{array}$ & 3 \\
\hline 3 & Acción preventiva. & 8.5 .3 & 4.5 .3 & $\begin{array}{l}\text { No conformidad, acción } \\
\text { correctiva y acción } \\
\text { preventiva. }\end{array}$ & 3 \\
\hline
\end{tabular}

El costo aproximado de la certificación para una IES considerada de tamaño pequeña (menos de 50 personas involucradas en los procesos certificados) es del orden de entre $\$ 8,000$ y $\$ 12,000$ dólares estadounidenses, que incluye auditorias de seguimiento, de certificación y el certificado valido por tres años. Bajo esta consideración el costo de certificación en México de los 322 SGC en IES bajo ISO 9001, no es menor a 2.5 millones de dólares estadounidenses.

Nivel de integración de ISO 9001 e ISO 14001. Al analizar el contenido de los estándares y partiendo de un SGC bajo ISO 9001:2008, se encuentra que 77 requisitos, es decir el 56.2\% de los 137 requisitos de ISO 9001 son integrables con los 62 de ISO 14001. Por otra parte 45 requisitos de ISO 14001, es decir el $72.5 \%$ son integrables con ISO 9001 tal como se observa en la Tabla 4. 
Tabla 4: Porcentaje de requisitos integrables entre ISO 9001:2008 e ISO 14001:2004.

\begin{tabular}{|l|c|c|}
\hline \multicolumn{1}{|c|}{ Estándar/Requisitos } & ISO 9001:2000 & ISO 14001:2004 \\
\hline Requisitos & 137 & 62 \\
\hline Requisitos integrables & 77 & 45 \\
\hline Porcentaje & $56.2 \%$ & $72.5 \%$ \\
\hline
\end{tabular}

Procedimientos integrales de gestión. En la Tabla 5 se proponen los procedimientos que debe de contener el Sistema Integral de Gestión, de los cuales 7 son procedimientos integrales, 6 procedimientos específicos para la norma ISO 14001:2004 y 3 son propuestos para la operación de una IES, dando un total de 16 procedimientos en lugar de 24 si se manejaran de manera separada; es decir 8 procedimientos menos, simplificando la documentación y ahorrando tiempo y dinero.

Tabla 5. Procedimientos propuestos; (o) procedimiento obligatorio, (r) Procedimiento recomendado para las IES, (i) Procedimiento integrado, (e) Procedimiento especifico para ISO 14001:2004.

\begin{tabular}{|c|c|c|}
\hline $\begin{array}{l}\text { Procedimientos de un } \\
\text { sistema de gestión de la } \\
\text { calidad bajo ISO 9001:2008 }\end{array}$ & $\begin{array}{l}\text { Procedimientos de un } \\
\text { sistema de gestión ambiental bajo } \\
\text { ISO 14001:2004 }\end{array}$ & $\begin{array}{l}\text { Procedimientos de un } \\
\text { Sistema Integral de } \\
\text { Gestión }\end{array}$ \\
\hline Control de documentos (o) & Control de documentos (o) & Control de documentos (i) \\
\hline Control de registros (o) & Control de registros (o) & Control de registros (i) \\
\hline $\begin{array}{l}\text { Competencia, formación y } \\
\text { toma de conciencia }(r)\end{array}$ & $\begin{array}{c}\text { Competencia, formación y toma de } \\
\text { conciencia (o) }\end{array}$ & $\begin{array}{c}\text { Competencia, formación y toma } \\
\text { de conciencia (i) }\end{array}$ \\
\hline Auditorias internas (o) & Auditorias internas (o) & Auditorias internas (i) \\
\hline Acciones correctivas (o) & Acciones correctivas (o) & Acciones correctivas (i) \\
\hline Acciones preventivas (o) & Acciones Preventivas (o) & Acciones preventivas (i) \\
\hline $\begin{array}{c}\text { Control de producto / servicio } \\
\text { no conforme (o) }\end{array}$ & Control de no conformidades (o) & Control de no conformidades (i) \\
\hline Prestación del servicio (r) & & Prestación del servicio (r) \\
\hline Inspección y prueba (r) & Aspectos ambientales (o) & $\begin{array}{l}\text { Aspectos ambientales / } \\
\text { significativos (e) }\end{array}$ \\
\hline \multirow[t]{7}{*}{$\begin{array}{c}\text { Reparación o baja de equipo no } \\
\text { conforme }(r)\end{array}$} & Requisitos legales (o) & Requisitos legales (e) \\
\hline & Comunicación (o) & Comunicación (e) \\
\hline & Control operacional (o) & Control operacional (e) \\
\hline & Respuesta a emergencias (o) & Respuesta a emergencias (e) \\
\hline & $\begin{array}{l}\text { Seguimiento y medición del } \\
\text { desempeño ambiental (o) }\end{array}$ & $\begin{array}{l}\text { Seguimiento y medición del } \\
\text { desempeño ambiental (e) }\end{array}$ \\
\hline & $\begin{array}{l}\text { Aspectos ambientales significativos } \\
(\mathrm{o})\end{array}$ & Inspección y prueba (r) \\
\hline & & $\begin{array}{l}\text { Reparación o baja de equipo no } \\
\text { conforme }(r)\end{array}$ \\
\hline
\end{tabular}

\section{DISCUSIÓN}

En México, la necesidad por contar con sistemas de gestión certificados, comenzó a principios de la década de los 90's por razones meramente comerciales, ya que el mercado requería proveedores confiables, es así que las empresas de transformación (mayormente de exportación), implementaron sistemas de gestión de la calidad principalmente bajo ISO 9001 y del medio ambiente bajo ISO 14001 entre otras. Posteriormente y bajo esta inercia natural de competitividad, las empresas de servicios y sectores como el gubernamental y el sector educativo, comenzaron a implantar también sistemas de gestión, principalmente de la calidad. Esta implementación se ha 
dado mayormente en servicios estratégicos y de apoyo dentro de las IES. Al respecto Srikanthan y Dalrymple (2005), indican que en el caso de las herramientas de calidad, éstas pueden ser aplicables a las partes de una Universidad con funciones similares a la industria como son las funciones administrativas y de apoyo. Esto concuerda con lo manifestado por Cidad (2004), quien observa en España que la mayoría de los certificados en Universidades han sido sobre procesos de apoyo y que debería ser contemplado como objeto primario de certificación los procesos estratégicos y básicos, sobre todo estos últimos, pues sobre ellos descansa mayoritariamente la tarea de añadirle valor al estudiante.

La facilitación al cambio y la innovación en las instituciones académicas para implementar exitosamente la gestión de la calidad, abarca la gestión de perspectivas de calidad de las partes interesadas tanto internas como externas a las IES comentan Becket y Brookes (2006); y es que aunque no se tratan de procesos similares, debemos de tener en cuenta lo que ha funcionado en la industria, y en la medida de lo posible adecuarlo a las actividades de las IES; similar a lo comentado por Venkatraman (2007), quien menciona que el éxito de herramientas de la gestión total de la calidad en la educación superior, depende de las lecciones trazadas por la aplicación en la industria.

Al revisar el número de certificados ISO 9001, observamos que la gestión de la calidad en las IES es una realidad, sin embargo, la gestión ambiental es actualmente una necesidad, lo que nos lleva a buscar formas que han tenido éxito al gestionar tanto la calidad como el cuidado al medio ambiente en las actividades diarias. En este sentido los sistemas integrales de gestión (SIG) brindan una excelente oportunidad para esta gestión. Jorgensen et al. (2006), comenta que en orden de crear ventajas competitivas para las organizaciones y contribuir a un desarrollo sustentable, los SIG deben expandirse para incluir la completa cadena de producción y todos las partes interesadas. Al respecto las IES forma parte de esta cadena al contribuir con personas y conocimiento, de ahí la importancia de predicar con el ejemplo.

Cidad (2004), comenta que algunas IES no han obtenido los beneficios esperados porque la curiosidad, la moda o incluso la exigencia del cliente, son motivaciones insuficientes si no se cuenta con algunas condiciones organizacionales previas facilitadoras del cambio y sobre las cuales se forja el logro de los beneficios que puede llegar a proporcionarle a la organización la implantación y mantenimiento del SGC; partiendo de las condiciones planteadas por Cidad (2004), se presentan las siguientes adecuadas a la gestión integral: Existencia de una cultura organizacional relativamente madura y, aunque implícitamente, orientada a la calidad y al medio ambiente, un clima laboral sano y proactivo, compromiso total de los líderes de la IES con el proyecto, orientación al trabajo en equipo, existencia de planificación básica-estratégica, un mínimo de procesos identificados y definidos, conciencia de mejora, evidenciada en algunas acciones concretas y entrenamiento de los integrantes.

Para continuar aportando en esta área se recomienda en trabajos futuros, realizar un estudio estadístico multivariado sobre las IES que han implementado SGC y SGA, además de realizar un estudio sobre el impacto real de estas certificaciones en los servicios educativos. Se recomienda, por otro lado, estudiar la posibilidad de integración de diferentes esquemas de acreditación de programas educativos, a los sistemas de gestión de calidad y del medio ambiente. Se recomienda a los docentes, estudiantes y profesionistas en general, que se preparen en estas disciplinas que son ya elementos necesarios del sector productivo y que puede marcar la diferencia al entender las ventajas de la integración de sistemas de gestión.

\section{CONCLUSIONES}

El reto de las Instituciones de Educación Superior implica desde siempre seguir siendo el ejemplo para la sociedad en que se encuentran, en este sentido el implementar sistemas como el que propone ISO 9001 para gestionar la calidad en los procesos claves, estratégicos y de apoyo de las IES juega un papel fundamental; sin embargo un buen desempeño ambiental es ya una necesidad en todas las IES, por lo que éstas pueden apoyarse en los sistemas de gestión ambiental como el que propone ISO 14001. Al respecto si ya se cuenta con un sistema de gestión de la calidad implementado, se tiene una excelente oportunidad de integrar un sistema de gestión ambiental a éste. 
Respecto al análisis de las normas ISO 9001:2008 e ISO 14001:2004, así como de los resultantes SGC y SGA se puede concluir lo siguiente:

A. La norma ISO 9001:2008 presenta un alto nivel de implementación en IES con respecto a ISO 14001:2004 en México.

B. Es mayormente factible la integración de un sistema de gestión ambiental a uno de gestión de la calidad ya implementado.

C. Las normas ISO 9001:2008 e ISO 14001:2004 presentan una plataforma alta de integración entre sí (56 \%), lo que puede ser aprovechado por las IES.

D. La derrama económica en México debido a la certificación en sistemas de gestión principalmente de la calidad y del medio ambiente en IES se considera alta.

E. No existe un consenso universal sobre la gestión de la calidad y del medio ambiente en IES.

\section{REFERENCIAS}

ACNielsen (en línea) International Standard Organization. Suiza (fecha de consulta 30 de Enero de 2009), http://www.iso.org/iso/survey2007.pdf (2009).

Alburquerque, P., B. Bronnenberg y C. Corbett; A spatiotemporal analysis of the global diffusion of ISO 9000 and ISO 14000 certification, Management Science: 53(3), 451-468 (2007).

Becket, N. y M. Brookes; Evaluating quality management in university departments, Quality Assurance in Education: 14(2), 123-142 (2006).

Cidad, E.; La gestión de la calidad en las organizaciones de educación superior: Aportación del enfoque de la Organización Internacional de Normalización (ISO), Revista Complutense de Educación: 15(2), 647-686 (2004).

Consejo de especialistas para la educación; Los retos de México en el futuro de la educación, Secretaria de educación pública. ISBN: 968-7485-25-6 (2006).

Complexus, Consorcio Mexicano de Programas Ambientales Universitarios para el Desarrollo Sustentable; "Declaratoria Complexus", VI Reunión anual del complexus, p1-5, Saltillo Coahuila, México (2006).

De-Olivera, J. y D. Coelho; The integration of the standars systems of quality management, environmental management and occupational health and safety management. International Journal of Production Research: 40(15), 3857-3866 (2002).

Douglas, A. y D. Glen; Integrated management system in small and medium enterprises, Total Quality Management: 4(11), 686-690 (2000).

Dounos, P. y G. Bohoris; "Exploring the interconnection of known TQM process improvement initiatives in Higher education with key CMMI concepts", Linköping University Electronic Press, ISSN [en línea], 2007, (fecha de consulta 28 de enero de 2009) http://www.ep.liu.se/ecp/026/109/ ecp0726109.pdf.

Houston, D.; TQM and higher education: a critical system perspective on fitness for purpose. Quality in Higher Education: 13(1), 3-17 (2007).

González, A.; "Modelo para lograr la integración de la gestión de la calidad, medio ambiente, y seguridad y salud ocupacional", 13va. Convención científica de ingeniería y arquitectura, Cujae, Cuba, (2006).

ISO, International Organization for Standardization, ISO-9001:2008/NMX-CC-9001-IMNC-2008. Instituto mexicano de Normalización y Certificación, México (2001). 
ISO, International Organization for standardization, ISO-14001:2004/NMX-SAA-14001-IMNC-2004. Instituto Mexicano de Normalization y Certification, Mexico (2004).

Jorgensen, T., A. Remmen y M. Mellado; Integrated management systems-three different levels of integration, Journal of Cleaner Production: 14(8), 713-722 (2006).

Karapetrovic, S. y W. Willborn; Integration of quality and environmental management systems. TQM Magazine: 101(3), 204-213 (1998).

Kit-Fai, P. y H. Ip-Kee; Integrating the Safety dimension into quality management systems: a process model, Total Quality Management: 13(3), 373-391 (2002).

Ortega, D.; "Las instituciones de educación superior ante el desafío del desarrollo sustentable". I Foro Nacional sobre la Incorporación de la Perspectiva Ambiental en la Formación Técnica y Profesional, p1-12, San Luis Potosí, México (2003).

Ortiz, B. y L. Nieto; "Complejidad, educación superior y medio ambiente: el caso del consorcio mexicano de programas ambientales universitarios para el desarrollo sustentable", I Foro Nacional sobre la Incorporación de la Perspectiva Ambiental en la Formación Técnica y Profesional, 1-10, San Luis Potosí, México (2003).

Potoski, M. y A. Prakash; Regulatory Convergence in Nongovernmental Regimes? Cross-National Adoption of ISO 14001 Certifications, The Journal of Politics: 66(3), 885-905 (2004).

Renzi, M. y L. Cappelli; Integration between ISO 9000 and ISO 14000: opportunities and limits, Total Quality Management: 11(4-6), 849-856 (2000).

Shaw, O.; "Hot Tips for implementing an integrated management system", Engineering Management: 10-13 (2004).

Srikanthan, G. y J. Dalrymple; Implementation of a Holistic Model for Quality in Higher Education, Quality in Higher Education: 11(1) (2005).

Suárez, E., "Anuario estadístico 2006-2007", ANUIESuperior [en línea], 2007, (fecha de consulta 10 de Diciembre de 2008), http://www.anuies.mx/servicios/e_educacion/docs/CUADROS\%20WEB

/Generales/Cuadros\%20Generales\%20REVISADOS\%202006-2007.xls

Venkatraman, S.; A framework for implementing TQM in higher education Programs, Quality Assurance in Education: 15(1), 92-112 (2007).

Wilkinson, G. y B. Dale; Models of management system standards: a review of the integration issues, International Journal of Management Reviews: 1(3), 279-298 (1999). 